\title{
Optimization of OMVPE-grown GaInP/GaAs quantum well interfaces
}

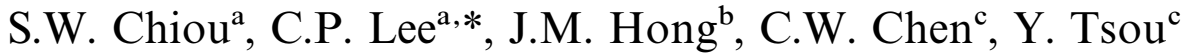 \\ ${ }^{a}$ Department of Electronics Engineering, National Chiao Tung University, 1001 Ta Hsueh Road, Hsinchu 30010, Taiwan, ROC \\ ${ }^{\mathrm{b}}$ Microelectronics Division, Hexawave Inc., Taiwan, ROC \\ ${ }^{\circ}$ Opto-Electronics and Systems Laboratories, Industrial Technology Research Institute, Hsinchu, Taiwan, ROC
}

Received 11 March 1999; accepted 14 June 1999

Communicated by G.B. Stringfellow

\begin{abstract}
A new and improved method of growing Al-free GaInP/GaAs quantum wells (QWs) is presented. We found that both interfaces are important for obtaining a high-quality quantum well. With an added $\mathrm{H}_{2}$ purge and $\mathrm{PH}_{3}$ preflow procedure, we have significantly improved the GaInP on GaAs interface by eliminating the unwanted dark line region at the interface. At the GaAs on GaInP interface, we added a thin GaAsP layer. This layer effectively prevents the In memory effect at the interface. As a result of this new growth procedure, a linewidth of $6.7 \mathrm{meV}$ (at $20 \mathrm{~K}$ ) for the quantum well emission was achieved. (C) 1999 Elsevier Science B.V. All rights reserved.
\end{abstract}

Keywords: High-quality quantum wells; GaInP/GaAs quantum wells; $\mathrm{PH}_{3}$

\section{Introduction}

Heterostructures with GaInP lattice-matched to GaAs offer many advantages over the AlGaAs/ GaAs system. This Al-free material is especially attractive in the production of laser diodes (LDs) [1], high electron mobility transistors (HEMTs) [2], and heterojunction bipolar transistors (HBTs) [3]. For example, a lower tendency to oxidize facilitates the fabrication of buried heterostructures and results in a more stable laser facets [4]. Very low surface recombination velocity and reduced DX centers make it suitable for manufacturing highspeed devices [5].

\footnotetext{
* Corresponding author.
}

The quality of the interface between GaInP and $\mathrm{GaAs}$ is a major concern for growing $\mathrm{GaAs} / \mathrm{GaInP}$ heterostructures. The most serious problem is a commonly observed unwanted GaInAsP intermediate layer existing at the GaAs/GaInP interface, resulting from the inter-mixing of the two constituent layers. Similar to the growth of $\mathrm{InP} / \mathrm{InGaAs}$ system, the problem is attributed to the As/P intermixing and the In memory effects $[4,6,7]$. Inserting a very thin $\mathrm{GaP}$ or AlGaAs layer between GaInP and GaAs [4,6] can improve the problem. But the lattice mismatch between GaAs and $\mathrm{GaP}$ is very large (about $4 \%$ ). Inserting a thin $\mathrm{GaP}$ layer between GaInP and GaAs may result in 3-D island growth and causes strain induced dislocations. Comparing to the lattice matched epitaxial growth, the interface roughness caused by this 
additional GaP interfacial layer may degrade the optical and electrical quality. Adding a thin lattice matched AlGaAs between GaInP and GaAs can yield a very good optical quality. But the electrical quality may suffer because of the high surface recombination rate of AlGaAs. In this paper, we report a new solution to overcome these problems.

\section{Experimental procedure}

The epitaxial layers used in this study were grown by a low-pressure organometallic vaporphase epitaxy (LP-OMVPE) system on $\left(\begin{array}{lll}1 & 0 & 0\end{array}\right)$ GaAs substrates oriented $10^{\circ}$ off to the $\left(\begin{array}{lll}0 & 1 & 1\end{array}\right)$ direction. The sources used were $\mathrm{AsH}_{3}, \mathrm{PH}_{3}, \mathrm{TMGa}$, TMIn, DMZn, and $\mathrm{Si}_{2} \mathrm{H}_{6}$. The pressure was 50 mbar and the growth temperature was $625^{\circ} \mathrm{C}$. The V/III ratios were 100 for GaAs and 200 for GaInP. To investigate the quality of the GaAs/ GaInP interface, we grew several quantum well structures with different added interfacial layers. The quantum well was a $7.5 \mathrm{~nm}$ GaAs layer sandwiched between two $100 \mathrm{~nm}$ GaInP barrier layers. After the layers were grown, they were characterized by photoluminescence (PL) at $20 \mathrm{~K}$.

Fig. 1a shows the $20 \mathrm{~K}$ PL spectrum of a conventional GaAs/GaInP QW without any added

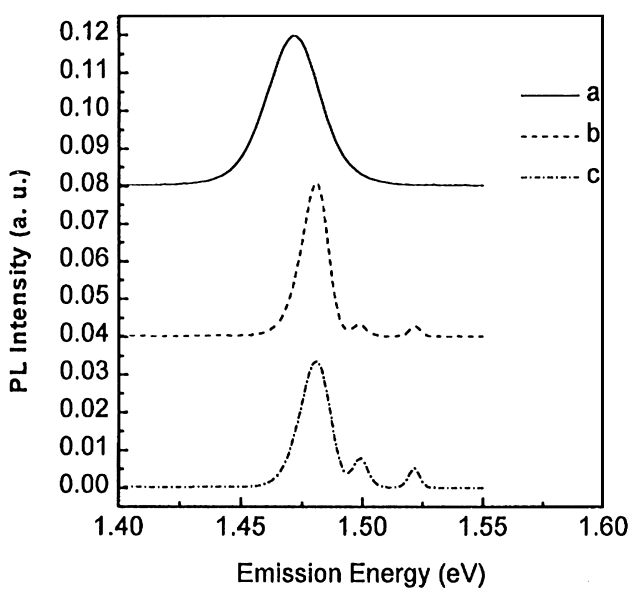

Fig. 1. PL spectra of GaAs/GaInP QW grown with (a) a normal gas switching sequence (b) an added $100 \mathrm{~s}_{2}$ purge at the inverse interface, and (c) an add $100 \mathrm{~s} \mathrm{H}_{2}$ purge and $20 \mathrm{~s}^{\mathrm{PH}_{3}}$ preflow at the inverse interface. interfacial layer. The result is similar to what has been reported $[4,6]$. No emission from the GaAs QW was observed. But instead, a broad peak at $1.47 \mathrm{eV}$, the so-called "anomalous band" emission, was observed. Previous studies have attributed the absence of QW emission to the bad quality of the inverse interface, where GaAs is grown after GaInP. We have performed TEM studies on $\mathrm{GaAs} / \mathrm{GaInP}$ double heterostructures and found that there was a dark line (a very narrow dark region) located at the interface where GaInP was grown on top of GaAs (it will be called the normal interface hereafter). The TEM image is shown in Fig. 2a. The interface is sharper than the inverse interface despite of the dark line. The dark line not only exists at the interface but also in the GaInP layer. As explained below, the dark line should be a GaInAsP compound. At the other (inverse) interface, there is an intermediate layer between GaInP and GaAs.

\section{Results and discussions}

\subsection{The GaInP on GaAs interface}

We attribute the formation of the dark lines at the normal interface to the As carry over effect. When the $\mathrm{PH}_{3}$ gas is switched on and $\mathrm{AsH}_{3}$ switched off, the residual $\mathrm{AsH}_{3}$ mixes with $\mathrm{PH}_{3}$ causing a dark line at the GaInP on GaAs interface. The second dark line, which is in the GaInP layer, is due to a different reason. During the growth of $\mathrm{GaInP}$, the high $\mathrm{PH}_{3}$ flow increases the desorption of As from the deposits on the reactor wall [7]. And this produces the second GaInAsP dark line in the GaInP layer. In order to improve the normal interface, we have employed a hydrogen purge and a $\mathrm{PH}_{3}$ pre-flow cycle during the growth of the interface. After the growth of GaAs, TMGa is switched off first. $\mathrm{AsH}_{3}$ is switched off after $10 \mathrm{~s}$ to ensure the completion of the surface reaction between the group III atoms and the group V atoms. Then we start the $100 \mathrm{~s} \mathrm{H}_{2}$ purge. After that we start the $\mathrm{PH}_{3}$ preflow for $20 \mathrm{~s}$. The purpose of this purge and the $\mathrm{PH}_{3}$ preflow cycle is to suppress the As carry over effect.

Fig. 1b shows PL spectrum of the GaInP/GaAs quantum well grown with a $100 \mathrm{~s}_{2}$ purge. No 

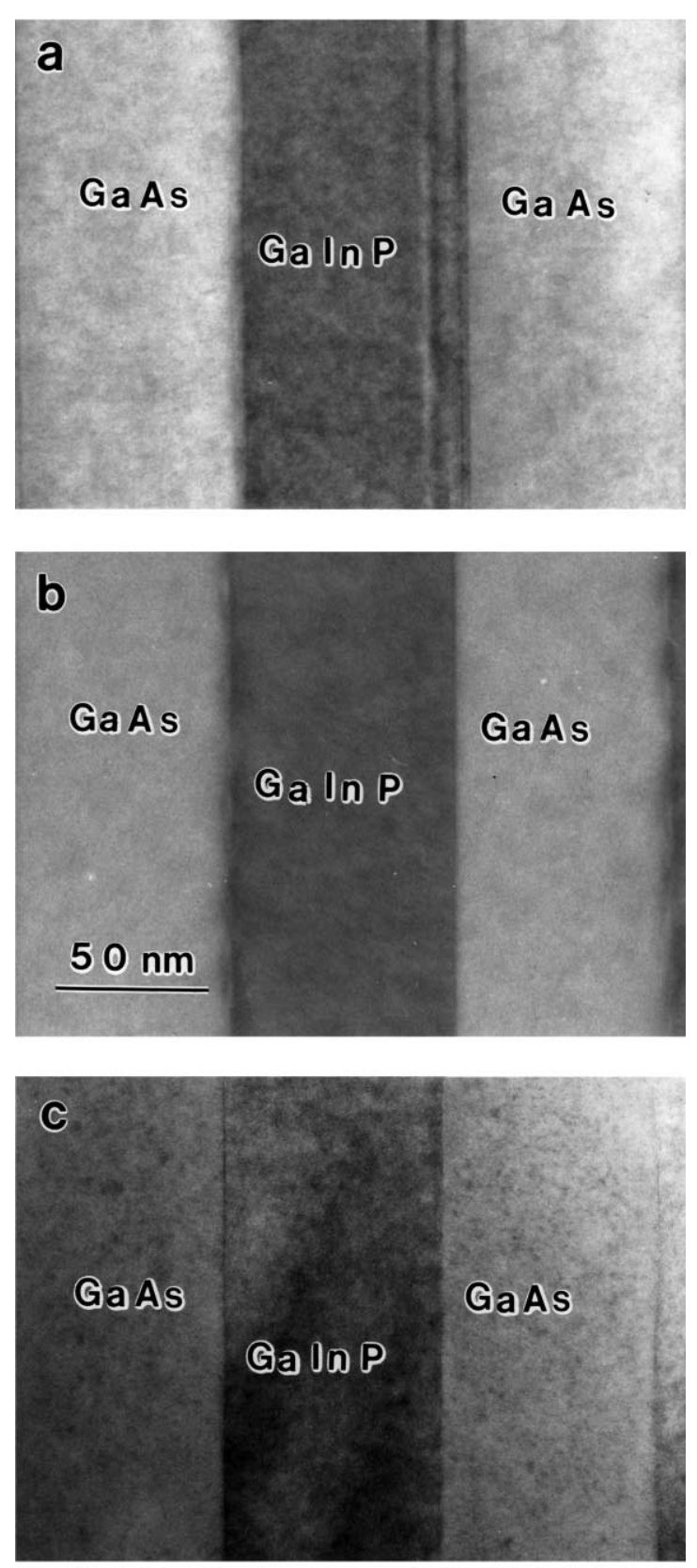

\section{Substrate}

Fig. 2. TEM photographs of the GaAs/GaInP double heterostructures grown with (a) a normal gas switching sequence, (b) an added $100 \mathrm{~s} \mathrm{H}_{2}$ purge and $20 \mathrm{~s} \mathrm{PH}_{3}$ preflow at the inverse interface and an added $1 \mathrm{~nm} \mathrm{GaP}$ at the normal interface, and (c) an added $100 \mathrm{~s} \mathrm{H}_{2}$ purge and $20 \mathrm{~s}^{\mathrm{PH}_{3}}$ preflow at the inverse interface and an added $1 \mathrm{~nm}$ GaAsP at the normal interface.
$\mathrm{PH}_{3}$ preflow was used. The 1.49 and $1.52 \mathrm{eV}$ emissions from the GaAs buffer layer start to show up. And the FWHM of the "anomalous band" becomes narrower and the energy is higher than that of the conventional sample.

$\mathrm{PH}_{3}$ preflow further improves the quality of the normal interface. The PL spectrum of a typical sample is shown in Fig. 1c, where a $\mathrm{PH}_{3}$ preflow of $20 \mathrm{~s}$ was used in addition to the $100 \mathrm{~s} \mathrm{H}_{2}$ purge. The intensities of the GaAs exciton peak from the buffer layer at $1.52 \mathrm{eV}$ and the $\mathrm{D}-\mathrm{A}$ peak at $1.50 \mathrm{eV}$ increase as compared to the anomalous band emission at $1.48 \mathrm{eV}$. But the QW emission is still absent.

\subsection{The GaAs on GaInP interface}

Having optimized the gas switching sequence for the $\mathrm{GaAs} / \mathrm{GaInP}$ normal interface, narrow GaAs $\mathrm{D}-\mathrm{A}$ and $\mathrm{X}-\mathrm{A}$ peaks can now be observed. But the $1.48 \mathrm{eV}$ deep emission peak still exists, which suggests that this deep emission might be associated with the other interface (the inverted interface). Earlier studies have shown that by applying a thin $\mathrm{GaP}$ layer at the GaAs on GaInP interface, the deep emission peak could be suppressed [4]. To clarify the role of $\mathrm{GaP}$ intermediate layer, we need to know the origin of the deep emission. As/P intermixing and In memory effect are possible causes for this interface problem. From the TEM result shown in Fig. 2a we know there is an intermediate layer at the interface. Since the $1.48 \mathrm{eV}$ emission has a lower energy than that of GaAs, this intermediate layer cannot be just caused by P carry over and the As/P intermixing, which would create a layer with a wider band gap than that of GaAs. So, the In memory effect has to play a very important role to create this low-energy emission interfacial layer. After the completion of the growth of GaInP layer, the residual TMIn in the reactor and the manifold will create an In-rich environment and will cause the growth of an InGaAs interfacial layer when the GaAs layer begins to grow.

If one adds a $\mathrm{GaP}$ layer at the interface, it can consume the residual TMIn and prevent the formation of InGaAs. However, the lattice mismatch between $\mathrm{GaP}$ and $\mathrm{GaAs} / \mathrm{GaInP}$ (around $4 \%$ ) could induce a large strain at the interface and causes degradation in material quality. Fig. 4a shows the 
PL spectrum of a GaAs/GaInP quantum well with an added $1 \mathrm{~nm} \mathrm{GaP}$ at the inverse interface. As expected, the low-energy anomalous band disappeared but the QW emission is very broad $(\mathrm{FWHM}=11 \mathrm{meV})$. This result is similar to what has been reported before $[4,8]$. The TEM image of such a quantum well is shown in Fig. 2b. We can see that the dark lines associated with the normal interface are no longer there because of the added $\mathrm{H}_{2}$ purge and $\mathrm{PH}_{3}$ preflow. However, at the other interface the junction is very rough. The rough interface is probably caused by the dislocations and 3-D island growth at the highly strained interface because of the added GaP.

To maintain the beneficial effect of the GaP interfacial layer but without the strain, Tsai et al. used a thin AlGaAs interfacial layer instead [6]. The AlGaAs layer did minimize the strain and as a result a narrow QW peak was obtained. However, this thin layer makes the material no longer Al-free and the problems commonly observed for Al-containing material may eventually degrade the device performance. In order to avoid the problems associated with the $\mathrm{GaP}$ and AlGaAs interfacial layers, we used a $1 \mathrm{~nm}$ GaAsP layer as an intermediate layer. The gas switching sequence used is shown in Fig. 3. Fukui et al. have shown that the $\mathrm{P}$ incorporation rate is very low at low growth temperatures [9]. In our case, we checked the lattice constant of a separately grown $1 \mu \mathrm{m}$ thick GaAsP layer by X-ray diffraction measurements. The lattice constant of bulk GaAsP layer is about $5.621 \AA$ A. So the phosphorus content in GaAsP is about $15 \%$. The lattice mismatch between GaAsP and GaAs is only $0.3 \%$. The PL spectrum of a QW grown this way is shown in Fig. 4b. A sharp and strong QW emission is observed at $1.546 \mathrm{eV}$. Most importantly, its FWHM is drastically reduced to $6.7 \mathrm{meV}$. Conventionally, during the growth of GaAs/GaInP, the group III flow is interrupted when the group $\mathrm{V}$ gas is switched over. But because of the In memory effect, an In-rich layer can still form at the interface. In our method, Ga species are supplied continuously without interruption. The large amount of TMGa reduces the concentration of the residual TMIn in the chamber and decreases the In memory effect. The amount of In remaining in the GaAsP layer is estimated to be only $10 \%$ by

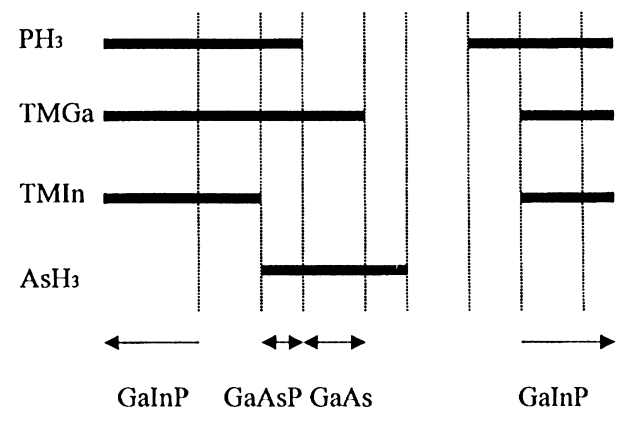

Fig. 3. The improved gas switching sequence for the growth of the GaAs/GaInP QWs at the normal interface, where the TMGa is not interrupted creating a $1 \mathrm{~nm}$ GaAsP intermediate layer.

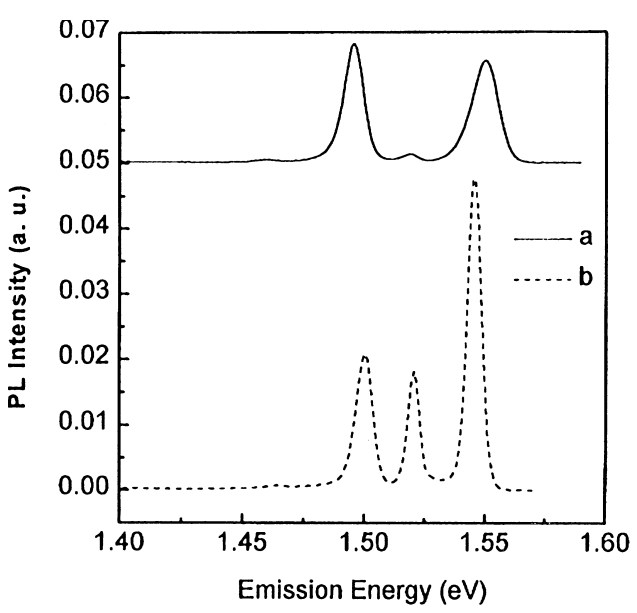

Fig. 4. PL spectra of a GaAs/GaInP QW with (a) an added $1 \mathrm{~nm}$ GaP layer at the normal interface, and (b) an added $1 \mathrm{~nm}$ GaAsP layer at the normal interface.

PL measurement. The lattice constant of the added GaAsP layer is very close to that of GaAs. So the strain is small and the dislocation problem does not happen. The TEM picture of a double heterostructure grown this way is shown in Fig. 2c. We see that both interfaces are perfect comparing with the other samples.

\section{Conclusions}

In summary, we have investigated the interface problem of $\mathrm{GaInP} / \mathrm{GaAs} \mathrm{QW}$. We found that both 
hetero-interfaces are important for a good QW. At the GaInP on GaAs interface, a long time $\mathrm{H}_{2}$ purge and $\mathrm{PH}_{3}$ preflow are necessary to eliminate the dark line at the interface. At the other interface (inverse interface), an added $1 \mathrm{~nm}$ GaAsP layer effectively eliminates the In memory effect. This layer is Al-free and has a lower lattice mismatch with GaAs (comparing with GaP). With this improved growth method, we have achieved a QW emission with a linewidth of only $6.7 \mathrm{meV}$.

\section{Acknowledgements}

We would like to thank C.W. Liang and S.Y. Wang for PL measurements and C.H. Baw for TEM measurements. This work was supported by the National Science Council of the Republic of China under contract NSC88-2215-E009-012.

\section{References}

[1] Z.L. Liau, S.C. Palmateer, S.H. Groves, J.N. Walpole, L.J. Missaggia, Appl. Phys. Lett. 60 (1992) 6.

[2] Y.J. Chan, D. Pavlidis, M. Razeghi, F. Omnes, IEEE Trans. Electron Dev. ED-37 (1990) 2141.

[3] T. Kobayashi, K. Taira, F. Nakamura, H. Kawai, J. Appl. Phys. 65 (1989) 4898.

[4] F.E.G. Guimaraes, B. Elsner, R. Westphalen, B. Spangenberg, H.J. Geelen, P. Balk, K. Heime, J. Crystal Growth 124 (1992) 199.

[5] J.M. Olson, R.K. Ahrenkiel, D.J. Dunlavy, B. Keyes, A.E. Kibbler, Appl. Phys. Lett. 55 (1989) 1208.

[6] C.Y. Tsai, M. Moser, C. Geng, V. Harle, T. Forner, P. Michler, A. Hangleiter, F. Scholz, J. Crystal Growth 145 (1994) 786.

[7] S.W. Ryu, W.G. Jeong, I. Kim, H.D. Kim, H.H. Kim, B.D. Choe, S.H. Park, J. Crystal Growth 179 (1997) 26.

[8] K. Uchida, T. Aral, K. Matsumoto, J. Appl. Phys. 81 (1997) 771.

[9] T. Fukui, N. Kobayashi, J. Crystal Growth 71 (1985) 9. 